\title{
Electronic structure and magnetic properties of the linear chain cuprates $\mathrm{Sr}_{2} \mathrm{CuO}_{3}$ and $\mathrm{Ca}_{2} \mathrm{CuO}_{3}$
}

\author{
H. Rosner, H. Eschrig, and R. Hayn \\ Max-Planck-Arbeitsgruppe 'Elektronensysteme', TU Dresden, D-01069 Dresden, Germany

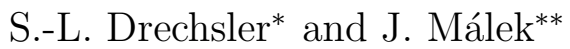 \\ Institut für Festkörper- und Werkstofforschung Dresden e.V., Postfach 270016, D-01171 Dresden, \\ Germany
}

(May 3, 2019)

\begin{abstract}
$\mathrm{Sr}_{2} \mathrm{CuO}_{3}$ and $\mathrm{Ca}_{2} \mathrm{CuO}_{3}$ are considered to be model systems of strongly anisotropic, spin-1/2 Heisenberg antiferromagnets. We report on the basis of a band-structure analysis within the local density approximation and on the basis of available experimental data a careful analysis of model parameters for extended Hubbard and Heisenberg models. Both insulating compounds show half-filled nearly one-dimensional antibonding bands within the LDA. That indicates the importance of strong on-site correlation effects. The bonding bands of $\mathrm{Ca}_{2} \mathrm{CuO}_{3}$ are shifted downwards by $0.7 \mathrm{eV}$ compared with $\mathrm{Sr}_{2} \mathrm{CuO}_{3}$, pointing to different Madelung fields and different on-site energies within the standard $p d$-model. Both compounds differ also significantly in the magnitude of the inter-chain dispersion along the crystallographical a-direction: $\approx 100$ $\mathrm{meV}$ and $250 \mathrm{meV}$, respectively. Using the band-structure and experimental data we parameterize a one-band extended Hubbard model for both materials which can be further mapped onto an anisotropic Heisenberg model. From the inter-chain dispersion we estimate a corresponding inter-chain exchange constant $J_{\perp} \approx 0.8$ and $3.6 \mathrm{meV}$ for $\mathrm{Sr}_{2} \mathrm{CuO}_{3}$ and $\mathrm{Ca}_{2} \mathrm{CuO}_{3}$, respectively. Comparing several approaches to anisotropic Heisenberg problems, namely the random phase spin wave approximation and modern versions of coupled quantum spin chains approaches, we observe the advantage of the latter in the reproduction of reasonable values for the Néel temperature $T_{N}$ and the magnetization $m_{0}$ at zero temperature. Our estimate of $J_{\perp}$ gives the right order of magnitude and the correct tendency going from $\mathrm{Sr}_{2} \mathrm{CuO}_{3}$ to $\mathrm{Ca}_{2} \mathrm{CuO}_{3}$. In a comparative study we also include $\mathrm{CuGeO}_{3}$.
\end{abstract}

71.15.Fv Atomic and molecular orbital methods

71.27.+a Strongly correlated electron systems

74.25.Jb Electronic structure

74.72.Jt other cuprates

75.30.Et Exchange and super-exchange

Typeset using REVTEX

75.50.El Antiferromagnetics 


\section{INTRODUCTION}

Initiated by the discovery of high- $T_{c}$ superconductivity in cuprate compounds, there is a renewed and growing interest in the electronic and magnetic properties of quasi one-dimensional (1D) structures near half band filling. Anionic quasi $1 \mathrm{D} \mathrm{CuO}_{3}$ chains of oxygen corner sharing $\mathrm{CuO}_{4}$ plaquetts are present in $\mathrm{Sr}_{2} \mathrm{CuO}_{3}$ and in $\mathrm{Ca}_{2} \mathrm{CuO}_{3}$ [1,2], and $\mathrm{CuO}_{2}$ chains of edge sharing plaquetts are present in $\mathrm{CuGeO}_{3}$ [3]. Moreover, the first two of these compounds can be regarded as 'parent' compounds of more complex structures as the double chain compound $\mathrm{SrCuO}_{2}$ (which, in notation $\mathrm{Sr}_{2} \mathrm{Cu}_{2} \mathrm{O}_{4}$, is obtained from $\mathrm{Sr}_{2} \mathrm{CuO}_{3}$ by replacing the $\mathrm{CuO}_{3}$ chain with a $\mathrm{Cu}_{2} \mathrm{O}_{4}$ double chain), and of a whole family of celebrated multi-leg ladder compounds $\mathrm{Sr}_{2 n} \mathrm{Cu}_{2 n+2} \mathrm{O}_{4 n+2}$ 泪. A detailed theoretical description of the 'parent' compounds is naturally a prerequisite for understanding all those materials. It is also desirable in view of the related two-dimensional (2D) cuprate structures of the high- $T_{c}$ materials [5 11], and particularly in view of possible dynamical stripe superstructures in the latter [12].

Experimentally, $\mathrm{Sr}_{2} \mathrm{CuO}_{3}$ and $\mathrm{Ca}_{2} \mathrm{CuO}_{3}$ are found to be the best realizations of the quasi $1 \mathrm{D}$ spin- $1 / 2$ antiferromagnetic Heisenberg model (AHM). Their Néel temperatures, $T_{N} \approx 5 \mathrm{~K}$ for $\mathrm{Sr}_{2} \mathrm{CuO}_{3}$ and $T_{N} \approx 9 \mathrm{~K}$ for $\mathrm{Ca}_{2} \mathrm{CuO}_{3}$, are very low compared to the intra-chain exchange integrals $J_{\|} \sim 0.2 \mathrm{eV}$, and the ordered moments $\left(<0.1 \mu_{\text {Bohr }}\right)$ are extremely small 13 15. The value $J_{\|}$ $=190 \mathrm{meV}$ for $\mathrm{Sr}_{2} \mathrm{CuO}_{3}$ [10 appears to be the record value of an exchange integral among all known quasi 1D antiferromagnets. The correct description of the physics of a magnetic quasi $1 \mathrm{D}$ system with a weak magnetic interchain interaction has recently attracted much theoretical attention, see, e.g., Refs. [16 [19]. For all these reasons, $\mathrm{Sr}_{2} \mathrm{CuO}_{3}$ has been announced to become a 'superstar' in the field of low-dimensional magnetism in near future [20].

In the work of Ami et al. [8], with the assumption of only a very weak dipolar inter-chain interaction constant $J_{\perp} \approx 0.01 \mathrm{meV}$ and on the basis of RPA spin wave theory, for $\mathrm{Sr}_{2} \mathrm{CuO}_{3}$ a Néel temperature below $0.03 \mathrm{~K}$ [21] was conjectured. At that time, due to resolution problems and because of the very small ordered moment $<0.1 \mu_{\mathrm{Bohr}}$, neutron diffraction on powders failed to detect antiferromagnetic ordering down to $1.5 \mathrm{~K}$. However, the relatively small inter-chain distances of 3.3 to $3.5 \AA$ suggest that direct inter-chain hopping, which leads to a much stronger kinematic exchange interaction, cannot be neglected. The discussion of consequences for the magnetic properties forms a main issue of the present paper.

While numerous band-structure calculations [22] and tight-binding parameterizations of one- and multi-band Hubbard model Hamiltonians 23] for the quasi 2D cuprate structures can be found in the literature, we are aware of only two band-structure calculations for a quasi 1D cuprate structure, both for $\mathrm{CuGeO}_{3}$ 24,25. On the basis of a wealth of available experimental data for $\mathrm{CuGeO}_{3}$ large efforts are currently directed to the parametrisation of phenomenological antiferromagnetic spin-1/2 Heisenberg models and exten- 
sions including frustration in the next-nearest neighbor intra-chain exchange 26 29]. To our knowledge, no parameterization on the level of Hubbard-type models has been undertaken as yet, although estimates within the Anderson impurity model [30] were pointing to strong correlation. The understanding of $\mathrm{CuO}_{3}$ chain substances is less developed, especially with respect to interchain interactions, and we shall present here a comparative analysis of both cases.

In contrast to the antiferromagnetic ordering of $\mathrm{Sr}_{2} \mathrm{CuO}_{3}$, the less anisotropic compound $\mathrm{CuGeO}_{3}$ exhibits a spin gap state below $T_{S P}=14.2 \mathrm{~K}$ which is accompanied by the occurrence of a period- 2 superstructure with a very small dimerization amplitude of $u_{0} \approx 0.007 \AA$. Therefore it has been interpreted as a spin-Peierls (SP) state. The SP state is supported by frustrated second neighbor exchange [26]. When doped with $\mathrm{Zn}$ for $\mathrm{Cu}$ or with $\mathrm{Si}$ for $\mathrm{Ge}$, also a coexisting Néel state has been found below 4.5K [31,32]. The thereby observed magnetic moment, for example of $0.23 \mu_{\mathrm{Bohr}}$ in $\mathrm{Cu}_{1-x} \mathrm{Zn}_{x} \mathrm{GeO}_{3}$, is significantly larger than the corresponding value of $0.06 \mu_{\mathrm{Bohr}}$ observed in $\mathrm{Sr}_{2} \mathrm{CuO}_{3}$ [13], suggesting a much larger anisotropy of the latter compound.

Within the frame of strongly anisotropic three-dimensional Heisenberg models, all considered compounds should be described by a dominating intrachain exchange coupling, a small exchange coupling in direction of the shortest inter-chain spacing which reduces the strong quantum fluctuations and provides a non-zero ordered magnetic moment in the ground-state, and a generally very small inter-chain coupling in the third direction to ensure a non-zero Néel temperature in accord with the Mermin-Wagner theorem. By applying the results of available theoretical approaches to the anisotropic two- and three-dimensional Heisenberg models [16 18, 33] one may extract phenomenological estimates of the exchange parameters for the chain cuprates, which then can be compared to results of electronic structure theory. Interestingly, different approaches yield significantly different predictions. The accurate determination of the Néel temperature of a strongly anisotropic Heisenberg magnet is still an unsolved and challenging theoretical task. We review here two frequently used approaches and compare their predictions from our estimates of exchange parameters with experimental data.

In the following Sec. II we present band-structure results for $\mathrm{Sr}_{2} \mathrm{CuO}_{3}$ and $\mathrm{Ca}_{2} \mathrm{CuO}_{3}$ which were obtained by applying a linear-combination-of-atomicorbitals scheme to self-consistently solve the Kohn-Sham equation with the local-density approximation to the exchange and correlation potential (LDALCAO). This approach provides us in a most natural way with tight-binding parameters. Using these results and experimental estimates for short-range correlation effects, in Sec. III a single $\mathrm{CuO}_{3}$ chain is represented by an extended Hubbard model and compared to the situation with $\mathrm{CuGeO}_{3}$. The magnitude of inter-chain exchange is estimated on this basis. In Sec. IV, estimates of Néel temperatures and ordered magnetic moments are derived by applying both standard RPA spin wave theory and modern quantum spin chain theory. The results are summarized in Sec. V, and an outlook is given. 


\section{BAND-STRUCTURE AND INTER-CHAIN TRANSFER}

The crystal structure of the isostructural compounds $\mathrm{Sr}_{2} \mathrm{CuO}_{3}$ and $\mathrm{Ca}_{2} \mathrm{CuO}_{3}$ is depicted in Fig. 1. Chains of oxygen corner sharing $\mathrm{CuO}_{4}$ plaquettes run along the b-direction. The in-chain $\mathrm{Cu}-\mathrm{O}$ bond-length is $1.96 \AA$ and practically the same in both compounds while the $\mathrm{Cu}-\mathrm{O}$ bond-lengths in c-direction differ: $1.95 \AA$ in $\mathrm{Sr}_{2} \mathrm{CuO}_{3}$ and $1.89 \AA$ in $\mathrm{Ca}_{2} \mathrm{CuO}_{3}$. The two inequivalent oxygen sites are referred to as chain oxygen and side oxygen in the following. The shortest inter-chain distance occurs in a-direction and differs substantially for both cases: $3.49 \AA$ in $\mathrm{Sr}_{2} \mathrm{CuO}_{3}$ and $3.28 \AA$ in $\mathrm{Ca}_{2} \mathrm{CuO}_{3}$.

The self-consistent LDA-LCAO method has been applied to both compounds with a minimum basis treating the Cu- $(4 s, 4 p, 3 d)$, O- $(2 s, 2 p)$, Sr$(5 s, 5 p, 4 d)$ and $\mathrm{Ca}-(4 s, 4 p, 3 d)$ orbitals as local valence basis states and the lower orbitals as core states. The crystal potential is calculated from overlapping spherical site densities. All basis states are calculated in the spherical site contribution to the crystal potential and recalculated in each iteration step. The valence basis orbitals have been compressed by an additional attractive potential to reduce the overlap among them [34]. Due to the relatively open crystal structure two empty spheres per unit cell have been introduced with empty sphere $s$ and $p$ orbitals at each site. For the exchange and correlation potential the parameterization of von Barth and Hedin was chosen and it has been calculated in atomic sphere approximation. We show in Fig. 2 the band-structure and in Fig. 3 the density of states (DOS) of $\mathrm{Sr}_{2} \mathrm{CuO}_{3}$. The corresponding results for $\mathrm{Ca}_{2} \mathrm{CuO}_{3}$ are similar. The quantitative differences between both compounds are discussed below. To check the LCAO-bandstructure by another method, we performed also calculations using the linear muffin-tin orbital (LMTO) approximation. We found no substantial differences, only the overall bandwidth of the whole $p d$ band complex was found to be somewhat smaller in the LMTO results (see numbers below).

As expected from simple chemical considerations of covalency, there is a single, well separated, nearly one dimensional, half-filled antibonding band crossing the Fermi level with large dispersion in b-direction (see Fig. 2). The width of this band is about $2.2 \mathrm{eV}$ (LCAO) or $2.0 \mathrm{eV}$ (LMTO) for both compounds. The characteristic quasi-1D van Hove singularities near the band edges are clearly seen in the DOS (see Fig. 3). A tight-binding analysis of the orbitals involved shows that in first approximation the $\mathrm{Cu} 3 d_{z^{2}-y^{2}}$ as well as the side oxygen $2 p_{z}$ and the chain oxygen $2 p_{y}$ orbitals are of direct relevance. Only a negligible admixture of $\mathrm{Cu} 4 s$ states can be detected near both edges of this antibonding half-filled band. Its weight as determined by the ratio of the corresponding areas under the DOS curves (see insert of Fig. 3) is relatively small (0.3 per cent for $\mathrm{Sr}_{2} \mathrm{CuO}_{3}$ ), but it increases to a weight of 2 per cent for the Ca compound.

The metallic behaviour of the LDA band-structure is in sharp contrast to the experimental observation of large optical gaps $\sim 2 \mathrm{eV}$ which are compara-

ble to the bandwidth obtained above. This points to the necessity of dealing 
explicitly with the strong on-site Coulomb repulsion at the copper-site. The experimental gap cannot be explained by a spin density wave since it is large and persists also above the Néel temperature $T_{N}$. Instead we have to anticipate the situation of a charge transfer gap between valence states of mostly oxygen character and a copper upper Hubbard band above the Fermi level.

In analogy to cuprates with $\mathrm{CuO}_{2}$ planes the construction of a multi-band, Hubbard-like model Hamiltonian would therefore be desirable. However, it is well known that such a Hamiltonian can be projected to an effective one-band picture which properly describes the low-energy physics [35,36]. The existence of a well isolated, one dimensional band in the present situation (shown in Fig. 4 in more detail) suggests such a possibility all the more. We assume that the parameters for the one band description can be determined by fitting the band of Fig. 4 to a dispersion of the form

$$
\varepsilon(\vec{k})=-2 t_{1, L D A} \cos \left(k_{y} b\right)-2 t_{2, L D A} \cos \left(2 k_{y} b\right)-2 t_{\perp} \cos k_{x} a
$$

which yields the values listed in the Table [37.

In Fig. 4 the dispersion in the a-direction is clearly visible as an energy increase with increasing $k_{x}$ by nearly the same amount both at the bottom and the top of the band. This dispersion is present through the band and gives a value of $t_{\perp}=25 \mathrm{meV}$. To be more accurate we determined $t_{\perp}$ from the dispersion at the Fermi level which is shown in the insert of Fig. 4. The corresponding dispersion for $\mathrm{Ca}_{2} \mathrm{CuO}_{3}$ is significantly larger, by a factor of 2.5 (2.0 in LMTO). The smaller lattice constant of $\mathrm{Ca}_{2} \mathrm{CuO}_{3}$ leads to an increase of the inter-chain overlap of the $\mathrm{Cu}-d$ and $\mathrm{O}-p$ basis orbitals, but this effect alone is too small to explain the strong enhancement. We have checked that the transfer in a-direction goes dominantly via the cation $\mathrm{Sr}$ and $\mathrm{Ca}$, respectively. The two-center Hamilton matrix elements between side oxygen and $\mathrm{Ca}$ are two times larger than the corresponding ones for Sr.

Dispersion in the c-direction is found within the level of accuracy of the band-structure calculations only $(\sim 5 \mathrm{meV})$. This practically missing dispersion in the c-direction indicates also that the inter-chain hopping in a-direction takes place horizontally only, with no zigzag contribution in (111) direction from side oxygen to side oxygen.

The comparison of the band structures of the $\mathrm{Sr}$ and the Ca compounds shows yet another interesting feature: namely, the bonding bands between $-8.5 \mathrm{eV}$ and $-11.5 \mathrm{eV}$ for $\mathrm{Sr}_{2} \mathrm{CuO}_{3}$ are shifted downwards by 0.6 to $0.7 \mathrm{eV}$ for the Ca compound indicating a significantly different Madelung field. For the same reason the side oxygen in the Ca-compound comes out more nega-

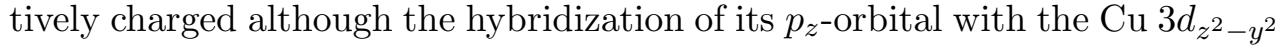
orbital is slightly increased due to the shortened bond-length. This different on-site energies may have an influence on the different charge transfer energies as discussed in the next section.

We also analyzed in the same manner (Eq. 1) the linearized augmented plane waves (LAPW) energy bands for $\mathrm{CuGeO}_{3}$ reported by Mattheiss [24]. $\mathrm{CuGeO}_{3}$ differs from the chain cuprates considered above in the structure 
of the chains. The $\mathrm{CuO}_{2}$ chains of oxygen edge-sharing $\mathrm{CuO}_{4}$ plaquetts of $\mathrm{CuGeO}_{3}$ result in a more complex highest antibonding band in which two O$p$ states per oxygen site hybridize with the $\mathrm{Cu}-d$ orbital. The more complex composition of the crystal also manifests in the band-structure. In particular, due to the sizeable inter-chain interaction mediated by Ge and due to the presence of two chains per unit cell there are two differently filled split-off antibonding bands. For our qualitative comparison with the above considered chains $\left(\mathrm{CuO}_{3}\right.$ chain), we replace them by one half-filled band for the sake of simplicity. The tight-binding parameters of $\mathrm{CuGeO}_{3}$ (Table) contain a significantly smaller nearest neighbor transfer integral $t_{1, L D A} \approx 0.25 \mathrm{eV}$ and an anomalously large next nearest neighbor integral [50] $t_{2, L D A} \approx 0.67 t_{1}=0.18$ $\mathrm{eV}$ estimated from Fig. 2 of Ref. [24]. The large difference of the transfer integrals $t_{1, L D A}$ between chains of corner sharing plaquetts and $\mathrm{CuGeO}_{3}$ should be related to the efficient $180^{\circ} \mathrm{Cu}-\mathrm{O}-\mathrm{Cu}$ hopping for the former $(\sigma p-d$ bond) compared with the inefficiency of non $\sigma p-d$ hopping (nearly $135^{\circ}$ $\left(45^{\circ}\right)$ ) for $\mathrm{CuGeO}_{3}$; for further details see Ref. 38]. This special structure explains also the relative large next nearest neighbor transfer integrals $t_{2}$ in $\mathrm{CuGeO}_{3}$ due to the involved effective $\sigma p_{z}-p_{z}$ hopping along the chain. The inter-chain hopping $t_{\perp}$ can be deduced from the dispersion in the b-direction of the LAPW energy bands of $\mathrm{CuGeO}_{3}$ and is of the same order as in $\mathrm{Sr}_{2} \mathrm{CuO}_{3}$ and $\mathrm{Ca}_{2} \mathrm{CuO}_{3}$.

Based on both the available experimental data and the band structure information obtained here, a semi-microscopic strong correlation model will be constructed which then can be mapped approximately onto a spin- $1 / 2$ Hamiltonian to describe the magnetic properties. This is the objective of the next section.

\section{MICROSCOPIC DESCRIPTION IN TERMS OF THE EXTENDED HUBBARD AND ANISOTROPIC HEISENBERG MODELS}

\section{A. General relations}

Here we parameterize the well-known extended Hubbard model for one single chain with hopping terms to first $\left(t_{1}\right)$ and second neighbors $\left(t_{2}\right)$ :

$$
\begin{aligned}
H= & -\sum_{m ; j=1,2 ; s} t_{j}\left(c_{m, s}^{\dagger} c_{m+j, s}+\text { h.c. }\right)+\frac{U}{2} \sum_{m ; s} c_{m, s}^{\dagger} c_{m, s} c_{m,-s}^{\dagger} c_{m,-s} \\
& +\sum_{m ; j=1,2} V_{j} n_{m} n_{m+j}-|K| \sum_{i} \vec{S}_{i} \vec{S}_{i+1},
\end{aligned}
$$

where $n_{m}=\sum_{s} c_{m, s}^{\dagger} c_{m, s}$ is the density operator and $s$ denotes the spin index. We included in (2) a small, but non-negligible direct ferromagnetic exchange which naturally appears if we map a multi-band, Hubbard-like Hamiltonian to a one band model [35]. Its necessity and its main effects will be discussed below. 
For the low-energy physics, at half-filling the extended Hubbard model (2) can be replaced to leading order in $t / U$ by a spin- $1 / 2$ Heisenberg chain. It includes also a second neighbor exchange $J_{2}$ [39] and reads

$$
\begin{aligned}
& H=J_{1} \sum_{i} \vec{S}_{i} \vec{S}_{i+1}+J_{2} \sum_{i} \vec{S}_{i} \vec{S}_{i+2}, \\
& J_{1}^{A F}=\frac{4 t_{1}^{2}}{U-V_{1}}, \quad J_{2}=\frac{4 t_{2}^{2}}{U-V_{2}},
\end{aligned}
$$

where the effective exchange integral $J_{1}$ of the spin-1/2 Heisenberg Hamiltonian of cuprates is reduced from the predominant antiferromagnetic superexchange part by the ferromagnetic contribution (2)

$$
J_{1} \approx-|K|+J_{1}^{A F} .
$$

Notice that within this approach $J_{2}$ yields a competitional (frustrated) character to the usually dominant short range antiferromagnetic correlations which are established by $J_{1}$. That term is especially important for $\mathrm{CuGeO}_{3}$.

The two main parameters $U$ and $t_{1}$ of the effective extended Hubbard model are directly related to the optical gap $E_{g}$ and the exchange integral between nearest neighbors $J_{1}$ which are experimentally accessible. The following analysis is considerably simplified if the materials of interest are in the strongly correlated limit $U>4 t$ and excitonic effects at zero momentum transfer $q$ are not very strong i.e. $U \gg t_{j}>V_{j} ; j=1,2$. The parameter sets derived below support such a point of view. We take into account the effect of the intersite Coulomb interaction $V_{1}$ by renormalizing the on-site correlation in the form $U_{e f f}=U-V_{1}$. Then we may use the optical gap $E_{g}$ obtained from the Bethe-Ansatz solution for the pure 1D Hubbard model given by Ovchinnikov 40],

$$
\begin{aligned}
E_{g} & =\frac{16 t_{1}^{2}}{U_{\text {eff }}} \int_{1}^{\infty} \frac{\sqrt{x^{2}-1} d x}{\sinh \left(2 \pi t_{1} x / U_{e f f}\right)} \\
& \approx U_{\text {eff }}-4 t_{1}+2 \ln 2 J_{1}^{A F} \quad \text { for } \quad U_{\text {eff }} \gg t_{1} ; \quad J_{1}^{A F}=4 t_{1}^{2} / U_{\text {eff }} .
\end{aligned}
$$

In the strong coupling case Eq. (5) can be transformed to the useful relation

$$
t_{1}=0.5 J_{1}^{A F}\left(1+\sqrt{E_{g} / J_{1}^{A F}+1-2 \ln 2}\right) .
$$

It has been assumed that the smaller parameters $t_{2}$ and $|K|$, i.e. the hopping to second neighbors and the ferromagnetic exchange in (2), have no substantial influence on the charge transfer gap (but $t_{2}$ enhances the spin gap in the spinPeierls state).

The presence of a weak second neighbor exchange can be approximately described in some cases by an effective renormalized nearest neighbor exchange integral 44, 45] 


$$
J=J_{1}-r J_{2} \approx J_{1}-J_{2}
$$

where $r=1$ according to Ref. 44] and $r=1.12$ according to Ref. 445. Recently, Stephan and Penc [46] predicted a strong narrow excitonic peak in the density-density response function $N(q, \omega)$ of the EHM in the strong coupling limit at the zone boundary $q=\pi / b$ :

$$
\omega_{e x}(\pi / b)=U-V_{1},
$$

provided $V_{1}>0$.

\section{B. Parameter assignment}

In principle, we can determine $U_{\text {eff }}$ and $t_{1}$ from the experimentally measured $E_{g}$ and $J$ values using Eqs. (47) which are presented graphically in Fig. 5. In the case of $\mathrm{Sr}_{2} \mathrm{CuO}_{3}$, very recently also the narrow excitonic peak at the zone-boundary (8) and with it $U_{\text {eff }}$ were determined experimentally 49.].

However, to the best of our knowledge, the available experimental information on all three systems is incomplete or contradicting each other. For instance, for $\mathrm{Ca}_{2} \mathrm{CuO}_{3}$ the charge gap determined from the maximum of $\Im m \varepsilon(\omega)$ is $E_{g}=2.1 \mathrm{eV}$ [5], but a direct measurement of the $J$ value from the magnetic susceptibility does not exist. Interpreting the midinfrared absorption as a phonon-assisted two magnon process a value of $J=255 \mathrm{meV}$ was reported [47]. For $\mathrm{Sr}_{2} \mathrm{CuO}_{3}$ the experimental $J$ values range from 140 to $260 \mathrm{meV}$ [9, 10,47]. In the following we shall use $190 \mathrm{meV}$ as a representative value. According to quite recent data for this system the one-dimensional charge transfer gap $E_{g}(\mathrm{Sr}) \approx 1.9 \pm 0.1$ [49 might be somewhat smaller as compared to the Ca compound. Strictly speaking, the optical absorption sets in already near $1.5 \mathrm{eV}$ 41,42,49. The elucidation of the observed broadening of the expected $1 \mathrm{D}$ van-Hove singularity in terms of the inter-chain interaction, quantum fluctuations, disorder, and/or excitonic and other many-body effects is a difficult problem beyond the scope of the present paper.

Taking this situation into account, we use the available experimental data and also our band-structure results to derive a consistent parameter set of (2) $\left(t_{1}, t_{2}, U_{e f f}, V_{1}\right.$ and $\left.|K|\right)$ for each of the three substances, separately. Vice versa, the demand of internal consistency weights the experimental information.

\section{1. $\mathrm{Sr}_{2} \mathrm{CuO}_{3}$}

Recent electron energy loss spectroscopy data of Neudert et al. 49. allow to determine $U_{\text {eff }}=3.15 \pm 0.1 \mathrm{eV}$ from the maximum of $\Im m \varepsilon(\omega)$ at the zone boundary (8). At the same time $E_{g}$ was measured to be $1.9 \pm 0.1 \mathrm{eV}$ from the data at small momentum. Similarly $E_{g}=1.92 \mathrm{eV}$ was found from the 
Raman resonance energy [11] observed for diagonal in-chain (yy) polarization only. Our aim is to derive values for the magnetic coupling constants from Eqs. (17) with the use of experimental values of $U_{\text {eff }}$ and $E_{g}$. Since it turns out that the derived $J$-values depend sensitively on $U_{\text {eff }}$ and $E_{g}$, we consider two sort of extreme cases. From Eq. (5) we obtain $t_{1}=0.410 \mathrm{eV}$ with $U_{\text {eff }}=3.15 \mathrm{eV}$ and $E_{g}=1.8 \mathrm{eV}$ (lower bound). That corresponds to $J_{1}^{A F}=213 \mathrm{meV}$. According to Eqs. (44, (7) that value has to be reduced by the frustrated next nearest neighbor exchange $J_{2}$ of about $12 \mathrm{meV}$ (corresponding to $t_{2}=100 \mathrm{meV}$ from our tight binding fit) and by the ferromagnetic contribution $|K|$ before it can be compared with the total experimental exchange integral $J=190 \pm 17 \mathrm{meV}$ [10]. Thus we can estimate a direct ferromagnetic exchange of $|K| \approx 11 \pm 17 \mathrm{meV}$. The slightly smaller $K$ value compared with $35 \mathrm{meV}$ for $\mathrm{La}_{2} \mathrm{CuO}_{4}$ obtained in Ref. [35] might be attributed to the shorter $\mathrm{Cu}-\mathrm{O}$ bond-length of $1.89 \AA$ for the latter compound. The so derived parameter set is listed in the Table. We derived a second parameter set with a considerable smaller value of $J_{1}$ by taking $E_{g}=1.95 \mathrm{eV}$ and $U_{\text {eff }}=3.25$ $\mathrm{eV}$ near the upper bounds of the experimental results. We obtain from (5) $t_{1}=0.394 \mathrm{eV}$ and correspondingly $J_{1}^{A F}=190 \mathrm{meV}$. Such a parameterization is compatible with the total exchange integral $147_{-9}^{+13} \mathrm{meV}$ [9] derived from the magnetic susceptibility data. We note that both parameterizations are incompatible with the large $J$ values of $246 \mathrm{meV} 48$ and $261 \mathrm{meV} 47$ derived from midinfrared optical absorption data (the small differences between the latter values arise mainly from the adopted phonon frequency of 70 and $80 \mathrm{meV}$, respectively, involved in the phonon-assisted absorption process) provided there is no sizeable ferromagnetic second neighbor exchange overcompensating the ferromagnetic nearest neighbor contribution $|K|$ and the antiferromagnetic next nearest neighbor superexchange $J_{2}=4 t_{2}^{2} / U_{\text {eff }}$. Anyhow, the elucidation of the microscopic origin of the apparent discrepancy between the magnetic susceptibility and the midinfrared optical absorption data analyzed in terms of the simple nearest neighbor spin-1/2 Heisenberg model remains a challenging problem.

\section{2. $\mathrm{Ca}_{2} \mathrm{CuO}_{3}$}

The slightly larger charge transfer gap of $2.1 \mathrm{eV}$ suggests also an enhanced $U_{e f f}$-value in comparison with the Sr-compound. That means that it is again difficult to find a reasonable parameterization which is compatible with the large $J$ value of $254 \mathrm{meV}$ from midinfrared absorption data. Due to the lack of experimental information on the magnetic susceptibility we use in the following our theoretical estimate of $160 \mathrm{meV}$ for the $J$ value of $\mathrm{Ca}_{2} \mathrm{CuO}_{3}$ [36]. Adding a ferromagnetic contribution of $|K| \approx 30 \mathrm{meV}$ (of the same order as for $\mathrm{La}_{2} \mathrm{CuO}_{4}$ ) and a frustrating $J_{2} \approx 10 \mathrm{meV}$ we may find $J_{1}^{A F}=200 \mathrm{meV}$. Of course, in the given case this should be considered as a very rough estimate. Then, together with $E_{g}=2.1 \mathrm{eV}$, we calculate from (6) $t_{1}=418 \mathrm{meV}$. According to (5) that corresponds to $U_{\text {eff }}=3.5 \mathrm{eV}$, showing the expected 
enhancement. On the level of the $p d$-model the reason for the enhanced effective on-site interaction should be traced back to a larger $\Delta \varepsilon_{p d} \approx U_{\text {eff }}$. It seems to be related to a Madelung effect caused by the difference in the lattice parameters of the $\mathrm{Sr}$ and $\mathrm{Ca}$ compound, respectively. This point of view is corroborated by our band-structure calculations discussed in the previous section: for $\mathrm{Ca}_{2} \mathrm{CuO}_{3}$ the distance between the half-filled antibonding band and the filled bonding bands is larger by $0.7 \mathrm{eV}$ compared to the $\mathrm{Sr}$ case.

\section{Inter-site Coulomb repulsion and comparison with $\mathrm{CuGeO}_{3}$}

According to the microscopic calculations of Geertsma and Khomski 38] the total nearest neighbor exchange integral of $\mathrm{CuGeO}_{3} J_{1}(\mathrm{Ge})=11.6 \mathrm{meV}$ can be decomposed into an antiferromagnetic contribution of $J_{1}^{A F}=30.4$ $\mathrm{meV}$ and into a relatively large ferromagnetic one of $|K|=18.8 \mathrm{meV}$. Let us stress again that the effective nearest neighbor transfer integral $t_{1}$ and the on-site interaction $U_{\text {eff }}$ are directly related to the antiferromagnetic part only. For $\mathrm{CuGeO}_{3}$ charge transfer gaps of $3.66 \mathrm{eV}$ [30] and $1.25 \mathrm{eV}$ 43. have been reported [50]. Using $E_{g}=3.7 \mathrm{eV}$ and $J_{1}^{A F}=30.4 \mathrm{meV}$, the main parameters of the extended Hubbard model come out as $t_{1}=0.187 \mathrm{eV}$ and $U_{\text {eff }}=4.34 \mathrm{eV}$. Interestingly, the latter value nearly perfectly coincides with the charge transfer energy $\Delta=4.2 \mathrm{eV}$ found out from the XPS data analyzed within the Anderson impurity model in Ref. [30]. Within a $p d$-model the significantly enhanced corresponding $\Delta \varepsilon_{p d}$ value should be attributed to the Ge-cations located near the $\mathrm{CuO}_{2}$ chain oxygens. This point of view is supported by the following observations. In compounds like $\mathrm{Sr}_{14} \mathrm{Cu}_{24} \mathrm{O}_{41}$ where the $\mathrm{CuO}_{2}$ chains under considerations are surrounded by earth alkaline cations the corresponding charge gap is reduced to about $2.8 \mathrm{eV}$ [51]. For that compound, $\Delta \varepsilon_{p d}$ as calculated within the ionic point charge model amounts to $3.7 \mathrm{eV}$ [52].

Comparing the data collected in the Table we suggest that $\mathrm{Ca}_{2} \mathrm{CuO}_{3}$ should be somewhat stronger correlated than its $\mathrm{Sr}$ counterpart. Without doubt, the most strongly correlated compound among them all is $\mathrm{CuGeO}_{3}$ having the smallest transfer integral $t_{1}(\mathrm{Ge})=0.187 \mathrm{eV}$ and the largest $U_{\text {eff }}(\mathrm{Ge})=4.34 \mathrm{eV}$. The large ratios $U_{\text {eff }} / t_{1}$ obtained in all three cases (7.7( $\mathrm{Sr}), 8.4(\mathrm{Ca})$, and $23(\mathrm{Ge}))$ justify a posteriori the use of Eq. (5).

The difference between $t_{1}$ and $t_{1, L D A}$ may be explained by a renormalization of the transfer integral $t_{1}$ by the inter-site Coulomb interaction $V_{1}$. Within the Hartree-Fock approximation, the correction due to $V_{1}$ leads to a renormalized effective hopping integral $t_{1}+p V_{1}$ with the bond order $p \sim 2 / \pi$. This renormalized hopping integral can be compared with $t_{1, L D A}$ where the inter-site Coulomb interaction is already partially taken into account. From $t_{1, L D A}=t_{1}+p V_{1}$ and the data of $t_{1}$ and $t_{1, L D A}$ given in the Table we may determine $V_{1}=0.21 \mathrm{eV}$ for $\mathrm{Sr}_{2} \mathrm{CuO}_{3}, V_{1}=0.16 \mathrm{eV}$ for $\mathrm{Ca}_{2} \mathrm{CuO}_{3}$, and $V_{1}=0.1$ $\mathrm{eV}$ for $\mathrm{CuGeO}_{3}$ (here $t_{1}=0.187 \mathrm{eV}$ as estimated above has been adopted). Thus, the inter-site Coulomb interactions $V_{1} \lesssim 0.2 \mathrm{eV}$ of all three compounds 
are quite close to each other and fulfill the relation $V_{1} \ll U$. Notice that these numbers for $V_{1}$ roughly agree with the corresponding $2 \mathrm{D}$-values $0.11 \mathrm{eV}$ or $0.17 \mathrm{eV}$ given in Refs. 35.53, respectively, and the estimate based on the fourband model for $\mathrm{CuO}_{3}$ chains [54]: $V_{1} \approx n_{d} n_{p} V_{p d}+n_{d}^{2}\left(V_{d d}-V_{2, d d}\right) \approx 0.23 \mathrm{eV}$, where typical occupation numbers $n_{d} \approx 0.7, n_{p} \approx 0.13$ and $V_{p d}=1.2 \mathrm{eV}$ for the copper oxygen inter-site Coulomb interaction and $V_{1, d d}=0.5\left(V_{2, d d}=0.25\right)$ $\mathrm{eV}$ for the (next) nearest neighbor copper copper inter-site Coulomb interaction have been taken.

The value for $t_{2}$ in the Table was either taken from the fit to the bandstructure data $\left(=t_{2, L D A}\right.$ for $\mathrm{Sr}_{2} \mathrm{CuO}_{3}$ and $\left.\mathrm{Ca}_{2} \mathrm{CuO}_{3}\right)$ or inferred from the experimentally known value for $J_{2}=4.3 \mathrm{meV}$ [26] for $\mathrm{CuGeO}_{3}$ using Eqs. (5).

\section{Inter-chain exchange}

Now, we would like to give a first estimate of the magnetic couplings between chains. The inter-chain exchange interaction $J_{\perp}$ in the a-direction for $\mathrm{Sr}_{2} \mathrm{CuO}_{3}$ and $\mathrm{Ca}_{2} \mathrm{CuO}_{3}$ (corresponding to the b-direction in $\mathrm{CuGeO}_{3}$ ) will be approximated by

$$
J_{\perp}=\frac{4 t_{\perp}^{2}}{U_{e f f}}
$$

where we assumed for simplicity the same inter-site Coulomb interaction $V_{1}$ within the chain and perpendicular to it. The corresponding values are listed in the Table. The discussion above about a possible direct ferromagnetic exchange which leads to a systematic reduction of exchange integrals suggests that these values should be considered as upper bounds. In the case of $\mathrm{CuGeO}_{3}$ the so determined $J_{\perp}=1 \mathrm{meV}$ can be compared with experimental data from neutron scattering [55,27] $J_{\perp} \approx 1.1 \mathrm{meV}$ showing a reasonable agreement. Notice that in the case of $\mathrm{Sr}_{2} \mathrm{CuO}_{3}$ our inter-chain interaction exceeds the dipolar interaction evaluated in Ref. [8] by two orders of magnitude.

The magnitude of the weakest interaction $J_{\perp, c}$ in the c-direction is difficult to estimate theoretically for several reasons. It was already discussed that a band-structure hopping integral cannot be given at present. In any case it may be expected that $J_{\perp, c}$ is smaller than the other exchange integrals by several orders of magnitude and is difficult to calculate in any case. Treating $J_{\perp, c}$ therefore as a purely phenomenological parameter in the following, we will use to be specific (if necessary) the same value for $\mathrm{Ca}_{2} \mathrm{CuO}_{3}$ as has been evaluated in Ref. [8] for $\mathrm{Sr}_{2} \mathrm{CuO}_{3}$, adopting the dipolar interaction for $J_{\perp, c} \approx 10^{-4} \mathrm{meV}$.

\section{SOME ASPECTS OF THE NÉEL STATE}

The magnetic properties of undoped cuprates (i.e. one hole per $\mathrm{Cu}$ site in the standard $p d$-model) are usually described by the anisotropic spin- $1 / 2$ antiferromagnetic Heisenberg model 


$$
H=\sum_{<i, j>} J_{i j} \mathbf{S}_{i} \mathbf{S}_{j}
$$

with $J_{i j}=J_{\|}\left(=J_{1}\right)$ for $(i j)$ beeing nearest neighbors in the chain direction (that is the b-direction for $\mathrm{Sr}_{2} \mathrm{CuO}_{3}$ and $\mathrm{Ca}_{2} \mathrm{CuO}_{3}$ ) and $J_{\perp}$ for nearest neighbor copper sites in a-direction (see Fig. 1). The weakest interaction will be denoted here by $J_{\perp, c}$. According to the results of the previous sections and from the experimental data we know that $\mathrm{Sr}_{2} \mathrm{CuO}_{3}$ and $\mathrm{Ca}_{2} \mathrm{CuO}_{3}$ are characterized by very anisotropic interaction strengths

$$
J_{\|} \gg J_{\perp} \gg\left|J_{\perp, c}\right|,
$$

the anisotropy being about three orders of magnitude for each inequality. Instead of the spin-Peierls system $\mathrm{CuGeO}_{3}$, in this section for comparison we will consider the doped compound $\mathrm{GeCu}_{1-x} \mathrm{Zn}_{x} \mathrm{O}_{3}(\mathrm{x}=0.034)$ [31,32] which shows antiferromagnetic order. This is an example for an anisotropic Heisenberg problem with weaker anisotropy than $\mathrm{Sr}_{2} \mathrm{CuO}_{3}$ and $\mathrm{Ca}_{2} \mathrm{CuO}_{3}$. For simplicity, we will use for $\mathrm{GeCu}_{1-x} \mathrm{Zn}_{x} \mathrm{O}_{3}$ the same parameters which were derived in the previous section for $\mathrm{CuGeO}_{3}$. We also neglect here the frustrated exchange $J_{2}$.

In the following we review several approaches for such anisotropic systems where quantum and thermal fluctuations become important. We will mention the usual spin wave approach in self-consistent random phase approximation (RPA-SWA) where all directions are treated on an equal and simple footing, and the coupled quantum spin chain approach (CQSCA) which involves a sophisticated treatment of the intra-chain direction and a mean-field treatment of the remaining perpendicular inter-chain interactions.

\section{A. RPA spin wave theory}

The RPA-SWA yields simple analytical expressions for the Néel temperature $T_{N}$ and for the magnetization $\left\langle S_{A}^{z}\right\rangle=m_{0}$ at zero temperature (see [8], 56 and references therein). Both quantities can be derived from the expression

$$
2 m(T)=\frac{1}{1+2 \psi}, \quad \psi=\frac{1}{N} \sum_{\vec{q}}\left(\frac{\omega_{0}}{\Omega(q)} \operatorname{coth}\left(\frac{\Omega(q)}{2 T}\right)-1\right),
$$

where

$$
\Omega(q)=\sqrt{\omega_{0}^{2}-\omega_{1}^{2}(q)}, \quad \omega_{1}=4 m(T) J_{\|}\left(\cos q_{y}+R \cos q_{x}+R_{c} \cos q_{z}\right),
$$

with $\omega_{0}=4 m(T)\left(J_{\|}+J_{\perp}+J_{\perp, c}\right), R=J_{\perp} / J_{\|}$and $R_{c}=J_{\perp, c} / J_{\|}$. We put $k_{B}=1$. The Néel temperature is defined by the condition of vanishing magnetization which yields

$$
2 T_{N}=J_{\|} / I\left(R, R_{c}\right)
$$


where

$$
I\left(R, R_{c}\right)=\frac{1}{\pi^{3}} \iiint_{0}^{\pi} \frac{d q_{x} d q_{y} d q_{z}}{R\left(1-\cos q_{x}\right)+R_{c}\left(1-\cos q_{z}\right)+\left(1-\cos q_{y}\right)} .
$$

Expanding $I\left(R, R_{c}\right)$ for $J_{\|} \gg J_{\perp} \gg J_{\perp, c}$ gives the approximate expression

$$
I\left(R, R_{c}\right)=\frac{0.66}{\sqrt{R}}\left(1+0.24\left(\ln \left(R / R_{c}\right)-R\right)\right),
$$

which determines the Néel temperature together with (14). The zero temperature magnetization $m_{0}$ is in the same limit given by

$$
m_{0}=\frac{0.303}{1-0.386 \ln (R)},
$$

where the small parameter $R_{c}$ turned out to be irrelevant. Notice that the RPA-description adopted reveals a vanishing magnetic moment in the $R \rightarrow$ 0 limit. Thus it differs from the ordinary spin-wave theory which yields a diverging expression $m_{0}=|0.5+(1 / \pi) \ln R|$ in the weak inter-chain coupling limit.

Let us now check the above expressions using the estimates of the last section and compare them with the experimental data. These data for $T_{N}$ and the magnetic moment $\mu^{e x p}=g_{L} m_{0}$ are given in the Table. In the following we will adopt a typical cuprate Landé factor $g_{L} \approx 2.1$ for $\mathrm{Cu}^{+2}$ [8]. Using the values $J_{\|}$and $J_{\perp}$ from the Table and $J_{\perp, c}=10^{-4} \mathrm{meV}$, we find $T_{N}^{S r}=38 \mathrm{~K}$, $T_{N}^{C a}=75 \mathrm{~K}, \mu^{S r}=0.20 \mu_{B}$ and $\mu^{C a}=0.26 \mu_{B}$ for the Sr and Ca compound, respectively. The ratio of the two experimental Néel temperatures agrees approximately with the RPA-SWA prediction

$$
T_{N}^{C a} / T_{N}^{S r} \approx \sqrt{J_{\|}^{C a} J_{\perp}^{C a}} / \sqrt{J_{\|}^{S r} J_{\perp}^{S r}} \approx 2
$$

where the logarithmic corrections in Eq. (13) can be neglected since they are not very important for the above ratio. However, the absolute values of $T_{N}$ within the RPA-SWA disagree with the experimental data. In the case of the more isotropic $\mathrm{GeCu}_{1-x} \mathrm{Zn}_{x} \mathrm{O}_{3}$ we find $\mu^{G e}=0.32 \mu_{B}$, i.e. a better agreement. But also here, the magnetic moment is overestimated by the RPA-SWA. In this case that may be ascribed to the effect of the frustrated second neighbor exchange.

For the $\mathrm{Sr}$ and Ca compounds one could try the opposite procedure using the given experimental data (including $J_{\|}$) to determine an "empirical" $J_{\perp}^{\text {emp }}$. Then one finds values for $J_{\perp}^{e m p}$ which are two (from $T_{N}$ ) or more than four (from $m_{0}$ ) orders of magnitude lower than those estimated in the previous section. This seems, therefore, to be unrealistic. Despite the fact that it gives the correct limits for $m_{0}$ both for $R \rightarrow 0$ and in the 2D isotropic case for $R \rightarrow 1$, the RPA-SWA seems to overestimate $m_{0}$ for large anisotropy $(R \ll 1)$ quite considerably. That points to the necessity for an improved method. In the case of smaller anisotropy (e.g. $R \sim 0.1$ like for $\mathrm{GeCu}_{1-x} \mathrm{Zn}_{x} \mathrm{O}_{3}$ ), the RPA-SWA seems to give more reliable results. 


\section{B. Coupled quantum spin chain approach (CQSCA)}

Adopting Schultz's interchain RPA-expression (Eq. (7) of Ref. [16]), we replace $J_{\perp} \rightarrow 0.5\left(J_{\perp}+J_{\perp, c}\right)$ as would be suggested by our strongly "orthorhombic" parameter regime $J_{\|} \gg J_{\perp} \gg J_{\perp, c}$. Then one arrives at

$$
m_{0}=\gamma \sqrt{R}
$$

where the proportionality factor $\gamma$ is 0.72 . (A similar factor $2 / \pi=0.637$, was obtained by Fukuyama et al. [57). The corresponding values for $\mu^{C S C}=$ $g_{L} m_{0}$ are listed in the Table.

Analogously, within these theories one expects $T_{N} \approx J_{\perp}$ [17], in particular, the slightly modified implicit expression for the transition temperature proposed by Schulz [16] reads

$$
T_{N}=\frac{2}{\pi} J_{\perp} \ln ^{1 / 2}\left(\Lambda J_{\|} / T_{N}\right)
$$

where $\Lambda \approx 5.8$.

From a principal point of view (Mermin-Wagner theorem), it is clear that Eq. (20) overestimates $T_{N}$ because it does not depend on $J_{\perp, c}$. However, if one assumes that its influence can be described by logarithmic terms like in Eq. (16) which then ensure a finite $T_{N}$, the relative changes might be quite small.

Like in the RPA-SWA, our estimated values for $J_{\perp}$ and the experimental $J_{\|}$lead to too large values for $T_{N}$ and $m_{0}$. But now, using the experimental $m_{0}$ and $J_{\|}$we can determine from Eq. (16) an "empirical" $J_{\perp}^{e m p}$ of the $\mathrm{CuO}_{3}$ chain compounds which is of a similar order of magnitude to our estimates. The value of $J_{\perp}^{e m p}$ is smaller by a factor between 2 and $3\left(\mathrm{Sr}_{2} \mathrm{CuO}_{3}\right.$, $\left.\mathrm{GeCu}_{1-x} \mathrm{Zn}_{x} \mathrm{O}_{3}\right)$, or $6\left(\mathrm{Ca}_{2} \mathrm{CuO}_{3}\right)$ compared to the theoretically estimated values (see Table). The Néel temperature can also be used to determine $J_{\perp}^{e m p}$ which gives similar values showing the internal consistency of the CQSCA. But one should keep in mind that Eq. (18) does not fulfill the Mermin-Wagner theorem. In that respect we note here an alternative approach to the strongly anisotropic Heisenberg model (Castro Neto and Hohn [33]) in which the Néel temperature was found to depend linearly on $J_{\perp, c}$. Naturally, the elucidation of the correct description as to how this smallest interaction parameter does affect the finite temperature properties remains a challenging problem. Without its generally accepted solution it makes no sense to discuss the absolute values of the Néel-temperature beyond an order of magnitude accuracy.

One possible explanation for the reduction of $J_{\perp}^{e m p}$ in comparison with our estimated $J_{\perp}$ could be the proximity of a spin-Peierls state. Phase fluctuation effects beyond the mean-field inter-chain approach used in deriving Eq. (19) can then become quite important. Following the renormalization group approach of Wang 19 for a plane of weakly interacting chains at $T=0$, one finds a strongly renormalized magnetization which can be traced back to a renormalized exchange integral. If that is true, one should expect $\mathrm{Ca}_{2} \mathrm{CuO}_{3}$ 
to be much closer to the SP phase transition point than $\mathrm{Sr}_{2} \mathrm{CuO}_{3}$. Furthermore for small exchange integrals compared with the phonon frequency $(\sim 10$ to $20 \mathrm{meV}$ ), phonon exchange gives rise to a quasi-instantaneous interaction between localized spins, leading to a renormalization $J \rightarrow J_{\text {eff }}<J$ [59].

Another possible origin for the difference between $J_{\perp}$ and $J_{\perp}^{e m p}$ might be our simple procedure to estimate $J_{\perp}$ based on the extended Hubbard model. It was already mentioned that such a procedure has the tendency to overestimate the exchange integrals which becomes already apparent for $J_{\|}$. Last not least, one should keep in mind an uncertainty of the band-structure methods with respect to transfer integrals as small as in the considered case. The replacement of the full potential in the region in between the chains by empty spheres as explained in Sec. II might effect the transverse tails of the Wannierfunctions which determine the value of the transfer integral $t_{\perp}$. A similar effect is expected if the exchange and correlational potential is supplemented with gradient terms reflecting the strong change in the electron density moving from one chain to its neighbors. Anyhow, one would expect roughly the same accuracy for the $\mathrm{Sr}$ and the Ca compound. In this context the much stronger deviation of the magnetic moment of the $\mathrm{Ca}$ compound might be related to somewhat reduced accuracy of Eq. (16) in less anisotropic cases.

\section{SUMMARY}

Band-structure calculations for $\mathrm{Sr}_{2} \mathrm{CuO}_{3}$ and $\mathrm{Ca}_{2} \mathrm{CuO}_{3}$ show in addition to the expected large dispersion along the chain direction also a remarkable dispersion in the crystallographic a-direction, i.e. perpendicular to the plane containing the corner-shared $\mathrm{CuO}_{4}$ plaquettes which form the $\mathrm{CuO}_{3}$ chains. The corresponding inter-chain transfer gives rise to antiferromagnetic exchange integrals $J_{\perp}$ in the meV range. Together with a small dipolar exchange in the third direction $J_{\perp, c}$ it explains the antiferromagnetic order in terms of an anisotropic Heisenberg model. The larger value of $J_{\perp}$ for $\mathrm{Ca}_{2} \mathrm{CuO}_{3}$ corresponds with the larger Néel temperature and the larger magnetic moment 15 in comparison with $\mathrm{Sr}_{2} \mathrm{CuO}_{3}$. However, our rough estimation of exchange integrals, based on the one band assumption, seems to overestimate the difference between both substances. $\mathrm{CuGeO}_{3}$ is different from these two substances by a much smaller exchange in chain direction and a comparable large frustration parameter which suppresses the antiferromagnetic state and stabilizes the spin Peierls state. The smaller anisotropy becomes apparent in $\mathrm{GeCu}_{1-x} \mathrm{Zn}_{x} \mathrm{O}_{3}$ where a Néel state was found with significantly larger magnetic moments than in $\mathrm{Sr}_{2} \mathrm{CuO}_{3}$ or $\mathrm{Ca}_{2} \mathrm{CuO}_{3}$.

The copper oxygen chains of the three compounds under consideration can be described within an extended Hubbard model supplemented by ferromagnetic contributions to the nearest neighbor exchange integral. For the $\mathrm{Ca}$ and $\mathrm{Sr}$ compounds excitonic effects in the limit $q \rightarrow 0$ are expected to be weak due to small inter-site Coulomb interaction $V_{1}$. But near the zone boundary $q \approx \pi / b$ strong excitonic effects are expected in the framework of the theory 
developed recently by Stefan and Penc 46]. If our proposed parameterization is correct, for $\mathrm{Ca}_{2} \mathrm{CuO}_{3}(\approx 3.5 \mathrm{eV})$ and $\mathrm{CuGeO}_{3}(\approx 4.3 \mathrm{eV})$ these excitonic peaks should be observed at higher energies than for $\mathrm{Sr}_{2} \mathrm{CuO}_{3}(3.15 \mathrm{eV})$.

The LDA band structure calculations yield useful insights into important material dependent parameters as inter-chain electron transfer and tendencies of the crystal field (Madelung ) potential, varying from one substance to the other, albeit that the estimate of the on-site and inter-site Coulomb interaction requires more sophisticated methods such as LDA-calculations with local constraint.

According to our findings, $\mathrm{Ca}_{2} \mathrm{CuO}_{3}$ is expected to be a typical $1 \mathrm{D}$ charge transfer insulator analogously to the $2 \mathrm{D}$ model system $\mathrm{Sr}_{2} \mathrm{CuO}_{2} \mathrm{Cl}_{2}$ although the inter-chain interaction is intermediate between the strongly correlated $\mathrm{CuGeO}_{3}$ and the most $1 \mathrm{D} \mathrm{Sr}_{2} \mathrm{CuO}_{3}$. Possibly, the latter system is the weakest correlated one of the three. The compounds $\mathrm{Sr}_{2} \mathrm{CuO}_{3}$ and $\mathrm{Ca}_{2} \mathrm{CuO}_{3}$ offer also the opportunity to study in detail the effect of the inter-chain interaction provided it can be changed in a controlled way. Indeed, the study of the magnetic properties of the alloy system $\mathrm{Sr}_{2-x} \mathrm{Ca}_{x} \mathrm{CuO}_{3}$ gives an interesting possibility to change continuously the magnitude of the inter-chain coupling. This is also interesting from the theoretical point of view since it gives a possibility to check in more detail sophisticated theories for weakly coupled quantum spin chains.

\section{ACKNOWLEDGMENTS}

Discussions with Profs. J. Fink, P. Fulde, D. Hone, H. Schulz, D. Johnston, and T. Van Oosten are acknowledged. Special thanks to Dr. M. S. Golden for discussions and a critical reading of the manuscript and to R. Neudert

providing us with EELS data for $\mathrm{Sr}_{2} \mathrm{CuO}_{3}$ prior to publication. One of us (J. M.) thanks the Max-Planck-Institut "Komplexe Systeme" Dresden, for hospitality during which part of the present work was performed. Finally, the Deutsche Forschungsgemeinschaft is also acknowledged for financial support (S.-L. D. and J. M.) . 


\section{REFERENCES}

* Author to whom all correspondence should be addressed, email-address: drechsler@ifwdresden.d400.de

** On leave from: Institute of Physics, Prague, Czech Republic.

1 C.L. Teske and H. Müller-Buschbaum, Z. Anorg. Allg. Chem. 371, 325 (1969).

2 M. Hjorth and J. Hyldtoft, Acta Chem. Scand. bf 44, 516 (1990).

3 M. Hase, I. Terasaki, and K. Uchinokura, Phys. Rev. Lett. 70, 3651 (1993).

4 K. Ishida, Y. Kitaoka, Y. Tokunga, S. Matsumoto, K. Asayama, M. Azuma,Z. Hiroi, and M. Takano, Phys. Rev. B 53, 2827 (1996).

5 SletY. Tokura, S. Koshihara, T. Arima, H. Takagi, S. Ishibashi, T. Ido, and S. Uchida, Phys. Rev. B 41, 11657 (1990).

$6 \quad$ M. Yoshida, S. Tajima, N. Kozhizusuka, S. Tanaka, S. Uchida, and S. Ishibashi, Phys. Rev. B 44, 11997 (1991).

7 A. Keren, L.P. Le, G.M. Luke, B.J. Sternlieb, W.D. Wu, Y.J. Uemura, S. Tajima, and S. Uchida, Phys. Rev. B 48, 12926 (1993).

8 T. Ami, M.K. Crawford, R.L. Harlow, Z.R. Wang, D.C. Johnston, Q. Huang, and R. Erwin, Phys. Rev. B 51, (1995).

$9 \quad$ S. Eggert, Phys. Rev. B 53, 5116 (1996).

10 N. Motoyama, H. Eisaki, and S. Uchida, Phys. Rev. Lett. 76, 3212 (1996);M. Takigawa, N. Motoyama, H. Eisaki, and S. Uchida, Phys. Rev. Lett. 76, 4612 (1996);.

11 O. Misochko, S. Tajima, C. Urano, H. Eisaki, and S. Uchida, Phys. Rev. B 53, R14 733 (1996).

12 V. Emery, S. Kivelson, and O. Zachar, sissa-preprint cond-mat/9610094 (1996).

13 K. Kojima, M. Larkin, B. Nachumi, Y. Uemura, H. Eisaki, M. Motoyama, S. Uchida, B. Sternlieb, and G. Shirane, Czechoslovak Journal of Phys. 46, Suppl. S4, 1945 (1996).

14 K. Yamada, J. Wada, S. Hosoya, Y. Endoh, S. Noguchi,

15 K. Kojima, Y. Fudamoto, M. Larkin, G. Luke, J. Merrin, B. Nachumi, Y. Uemura, N. Motoyama, H. Eisaki, S. Uchida, K. Yamada, Y. Endoh, S. Hosoya, B. Sternlieb, and G. Shirane, Phys. Rev. Lett. 78, 1787 (1997).

16 H.J. Schulz, Phys. Rev. Lett. 77, 2790 (1996).

17 I. Affleck, M. Gelfand, and R. Singh, J. Phys. A 27, 7313 (1994), Erratum 28, 1787 (1995), and sissa-preprint cond-mat/9408062 (1994).

18 I. Affleck, and B. Halperin, sissa-preprint cond-mat/9603078 (1996).

19 Z. Wang, Phys. Rev. Lett. 78, 126 (1997), cond-mat/9611129 (1996).

20 A. Tsvelik "Quantum Field Theory in Condensed Matter", Cambridge University Press, p. 264, 155 (1995).

21 Adopting the applicability of the RPA-spin wave theory and the value of the weak dipolar interchain interaction of about $0.01 \mathrm{meV}$ evaluated in [8] one would arrive at $T_{N}=6.2 \mathrm{~K}$, i.e. accidentily close to the experimental value, in sharp contrast to the stated $T_{N} \approx 0.028 \mathrm{~K}$, only. Using the more realistic interchain value of about $1 \mathrm{meV}$ obtained below, one arrives formally at $T_{N} \approx 29 \mathrm{~K}$. For a critical discussion of that approach see Sec. IV.

22 W. E. Pickett, Rev. Mod. Phys. 61, 433 (1989).

23 M. S. Hybertsen, E. B. Stechel, M. Schlüter, and D. R. Jennison, Phys. Rev. B 41, 11068 (1990).

24 L.F. Mattheiss, Phys. Rev. B 49, 14050 (1994).

25 Z. Popović, F. Vukajlović, and Z. Slivancanin, J. Phys. Condens. Mat. 7, 4549 (1995). 

mat/9610134 and Phys. Rev. B, 55, (1997), in press.

29 V. Muthukumar, C. Gros, W. Wenzel, R. Valentí, P. Lemmens, B. Eisener, G. Güntherodt, M. Weiden, C. Geipel, and F. Steglich, Phys. Rev. B 54,R9635 (1996); V. Muthukumar, C. Gros, R. Valentí, M. Weiden, C. Geipel, F. Steglich, P. Lemmens, M. Fischer, and G. Güntherodt, sissa-prep. cond-mat/9611018. F. Parmigiani, L. Sangaletti, A. Goldoni, U. del Pent
and G. Dhalénne, Phys. Rev. B, 55, 1459 (1997).

M. Hase, K. Uchinokura, R. Birgeneau, K. Hirota, and G. Shirane, J. Phys. Soc. Jap. 65, 1392 (1996).

M. Poirier, R. Beaudry, M. Castonguay, M. Plumer, G. Quirion, F. Razavi, A. Revcolevschi, and G. Dhalenne, Phys. Rev. B 52, R6971 (1995).

A.H. Castro Neto and D. Hone, Phys. Rev. Lett. 76, 2165 (1996), D. Hone and A. Castro Neto, J. of Superconductivity 10 in press. and M. Mostovoy, Proc. LT21 Prague 1996, Czech Journal of Physics, 46-Suppl. part 6, 3239 (1996).

H. Eschrig, Optimized LCAO Method (Springer-Verlag, Berlin, 1989). H.-B. Schüttler and A.J. Fedro, Phys. Rev. B 45, 7588 (1992).

S.-L. Drechsler, J. Malek, S. Zalis, and K. Rościszewski, Phys. Rev. B, 53, 11328 (1996); J. of Superconductivity 9, 439 (1996); ibid. 10 in press.

Strictly speaking, the fit of the dispersion along the a-direction requires more Fouriercomponents. However, their individual weight is small with respect to the interchain exchange. It can be expected that within a proper treatment of the local electron correlation the influence of far reaching hopping integrals should be reduced.

W. Geertsma and D. Khomskii, Phys. Rev. B 54, 3011 (1996); D. Khomskii, W. Geertsma, K. Schulten, I. Ohmine, and M. Karplus, J. of Chem. Phys. 64, 4442 (1976).

A. Ovchinnikov, Zh. Eksp. Teor. Fiz. 57, 2137 (1969) (or Sov. Phys. JETP 30, 1160 (1970)).

K. Okada, A. Kotani, K. Maiti, and D.D. Sarma, J. Jpn. 56, 1844 (1996)

K. Maiti, D.D. Sarma, T. Mizokawa, and A. Fujimori, Europhysics Lett. 37, 359 (1997).

I. Terasaki, R. Itti, N. Koshizuka, M. Hase, I. Tsukuda, and K. Uchinokura, Phys. Rev. B, 52, 295 (1995).

D. Gottlieb, M. Lagos, K. Hallberg, and C. Balseiro, Phys. Rev. B 43, 13668 (1991).

A. Fledderjohann and C. Gros, sissa-preprint cond-mat/9612013 (1996).

W. Stephan and K. Penc, Phys. Rev. B 54, R 17269 (1996).

H. Suzuura, H. Yasuhara, A. Furusaki, N. Nagaosa, and Y. Tokura, Phys. Rev. Lett. 76, 2579 (1996).

J. Lorenzana and R. Eder, Phys. Rev. B 55, R3358 (1997).

R. Neudert et al. to be published.

The attribution of sharp peaks near $1.25 \mathrm{eV}, 2.9 \mathrm{eV}$ and $3.66 \mathrm{eV}$ observed in $\operatorname{Im} \varepsilon(\omega)$ to the $d p$ charge transfer gap which is of interest here, to $d d$-transitions, and to Ge-states related interband transition is controverse.

T. Osafune, N. Motoyama, H. Eisaki, and S. Uchida, Phys. Rev. Lett. 78, 1980 (1997). 
Y. Mizuno, T, Tohyama, and S. Maekawa, J. Phys. Soc. Jap. to be published; sissa-preprint cond-mat/9612252 (1996).

53 L. Feiner, J. Jefferson, and R. Raimondi, Phys. Rev. B 53, 8751 (1996). S.-L. Drechsler, J. Malek, and H. Eschrig, Phys. Rev. B 55, 606 (1997).

55 M. Nishi, O. Fujita, J. Akimitsu, K. Kakurai, and Y. Fujii, Physica B 213 \& 214, 275 (1995).

N. Majilis, S. Selzer, and G. Strinati, Phys. Rev. B 45, 7872 (1992).

57 H. Fukuyama,T. Tanimoto, and M. Saito, J. of Phys. Soc. Jap. 65, 1182 (1996).

58 M. Honda, T. Shirata, K. Kindo, S. Sugai, T. Takeuchi, and H. Hori, J. of Phys. Soc. Jap. 65, 691 (1996).

59 H. Schulz in "Low Dimensional Conductors and Superconductors" (Eds. D. Jerome and L.G. Caron), NATO ASI series 155, p. 95, Plenum Press, New York (1987). 


\section{TABLES}

TAB. Model parameters for $\mathrm{Sr}_{2} \mathrm{CuO}_{3}, \mathrm{Ca}_{2} \mathrm{CuO}_{3}$ and $\mathrm{CuGeO}_{3}$. The LDALCAO derived tight binding parameters in the first group of rows are explained in Sec. II. The LDA-numbers for $\mathrm{CuGeO}_{3}$ are estimated from Figs. 2 of Ref. 24] and Ref. [25]. The second group contains experimental values (in the case of several date we prefer the underlined) which were used in addition to the band-structure information to estimate the corresponding parameters of the extended Hubbard model as well as the exchange integrals of the anisotropic Heisenberg-model (third group of rows). They are derived and discussed in Sec. III. The experimental magnetic moment $\mu^{e x p}$ (given together with $T_{N}$ in group IV) may be compared with $\mu^{C S C}$ derived from Eq. (19) using the experimental data for the in-chain exchange integrals $J_{\|}$and

our estimation of $J_{\perp}$. Vice versa, the experimental $\mu^{\exp }$ determines via the same Eq. (19) the empirical inter-chain exchange integrals $J_{\perp}^{e m p}$. 


\begin{tabular}{|c|c|c|c|c|}
\hline group & quantity & $\mathrm{Sr}_{2} \mathrm{CuO}_{3}$ & $\mathrm{Ca}_{2} \mathrm{CuO}_{3}$ & $\begin{array}{c}\mathrm{CuGeO}_{3} \\
\left(\mathrm{GeCu}_{1-x} \mathrm{Zn}_{x} \mathrm{O}_{3}\right)\end{array}$ \\
\hline 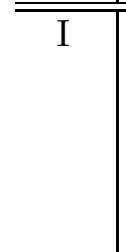 & $\begin{array}{c}t_{1, L D A} / \mathrm{meV} \\
t_{2, L D A} / \mathrm{meV} \\
t_{\perp} / \mathrm{meV}\end{array}$ & $\begin{array}{c}550 \\
100 \\
20 \text { to } 30\end{array}$ & $\begin{array}{c}520 \\
100 \\
50 \text { to } 65\end{array}$ & $\begin{array}{c}250 \\
81 \\
25 \text { to } 33\end{array}$ \\
\hline II & $\begin{array}{c}E_{g} / \mathrm{eV} \\
J / \mathrm{meV}\left(=J_{\|} / \mathrm{meV}\right)\end{array}$ & $\begin{array}{c}(1.8 \text { to } 1.9)^{\mathrm{a}, \mathrm{b}} \\
(140)^{\mathrm{f}},(\underline{190})^{\mathrm{g}},(260)^{\mathrm{h}}\end{array}$ & $\begin{array}{c}(2.1)^{\mathrm{c}} \\
(\underline{160})^{\mathrm{i}}(254)^{\mathrm{h}}\end{array}$ & $\begin{array}{l}(1.25)^{\mathrm{d}},(\underline{3.7})^{\mathrm{e}} \\
(\underline{11} \pm 1)^{\mathrm{j}},(22)^{\mathrm{k}}\end{array}$ \\
\hline III & $\begin{array}{c}t_{1} / \mathrm{meV} \\
t_{2} / \mathrm{meV} \\
U_{e f f} / \mathrm{eV} \\
V_{1} / \mathrm{eV} \\
|K| / \mathrm{meV} \\
J_{\perp} / \mathrm{meV}\end{array}$ & $\begin{array}{c}410 \\
100 \\
(3.15)^{b} \\
0.21 \\
11 \\
0.5 \text { to } 1.1\end{array}$ & $\begin{array}{c}419 \\
100 \\
3.5 \\
0.16 \\
30 \\
2.9 \text { to } 4.3\end{array}$ & $\begin{array}{c}187 \\
90 \\
4.34 \quad(4.2)^{e} \\
0.1 \\
19 \\
0.6 \text { to } 1,(1.1)^{l}\end{array}$ \\
\hline IV & $\begin{array}{c}T_{N} / \mathrm{K} \\
\mu^{e x p} / \mu_{B}\end{array}$ & $\begin{array}{c}(5)^{l} \\
(0.06 \pm 0.01)^{l}\end{array}$ & $\begin{array}{c}(8 \ldots 10)^{l} \\
(0.09 \pm 0.01)^{l}\end{array}$ & $\begin{array}{l}(4.5)^{m} \\
(0.23)^{j}\end{array}$ \\
\hline $\mathrm{V}$ & $\begin{array}{l}\mu^{C S C} / \mu_{B} \\
J_{\perp}^{e m p} / \mathrm{meV}\end{array}$ & $\begin{array}{c}0.08 \text { to } 0.11 \\
0.3 \pm 0.1\end{array}$ & $\begin{array}{c}0.19 \text { to } 0.24 \\
0.6 \pm 0.1\end{array}$ & $\begin{array}{c}0.35 \text { to } 0.45 \\
0.27\end{array}$ \\
\hline
\end{tabular}

${ }^{\text {a }}$ Raman resonance, Ref. [11]

b EELS, Ref. 49.

c opt. absorption, Ref. [5]

d XPS, Ref. 43.

e XPS, Ref. 30

${ }^{f}$ magn. suscept., Ref. 8,9]

g magn. suscept., Refs. 10

$\mathrm{h}$ midinfrared, Ref. 47

i theory, Ref. 36.

j INS, Raman, Refs. [27,29,31,45]

k Raman, Ref. 28]

${ }^{1} \mu \mathrm{SR}$, Ref. 15

m INS, Ref. 55 


\section{FIGURES}

FIG. 1 Crystal structure of $\mathrm{Sr}_{2} \mathrm{CuO}_{3}$

FIG. 2 LCAO energy-bands near the Fermi level for $\mathrm{Sr}_{2} \mathrm{CuO}_{3}$ along highsymmetry Brillouin-zone directions within the $\left(k_{x}, k_{y}\right)$ plane. The momenta are given in units of $(\pi / a, \pi / b)$. Strong dispersion can be seen along $(0,0)-(0,1)$ (b-direction, parallel to the $\mathrm{CuO}_{3}$ chains) whereas a small, but non-negligible dispersion in the perpendicular a-direction can be seen.

FIG. 3 Density of states $N(E)$ for $\mathrm{Sr}_{2} \mathrm{CuO}_{3}$. The insert shows the partial $\mathrm{Cu}$ $3 d$ and $4 s$ density of states of the nearly one-dimensional band crossing the Fermi level.

FIG. 4 Dispersion of the nearly one-dimensional band. The insert is for fixed $k_{y}=k_{y \text { Fermi }}=\pi / 2 b$.

FIG. 5 Dependence of the transfer integral $t_{1}$ (upper panel) and the on-site Coulomb interaction $U_{\text {eff }}$ (lower panel) of the Hubbard model according to its Bethe ansatz-solution vs. inchain superexchange integral $J_{1}^{A F}$ (lower panel) in the strong coupling limit for typical values of the optical gap $E_{g}$. The experimental values for $J_{1}^{A F}$ are depicted by arrows. They are determined from the total exchange integral $J$ adopting ferromagnetic and second neighbor contributions discussed in the text. 


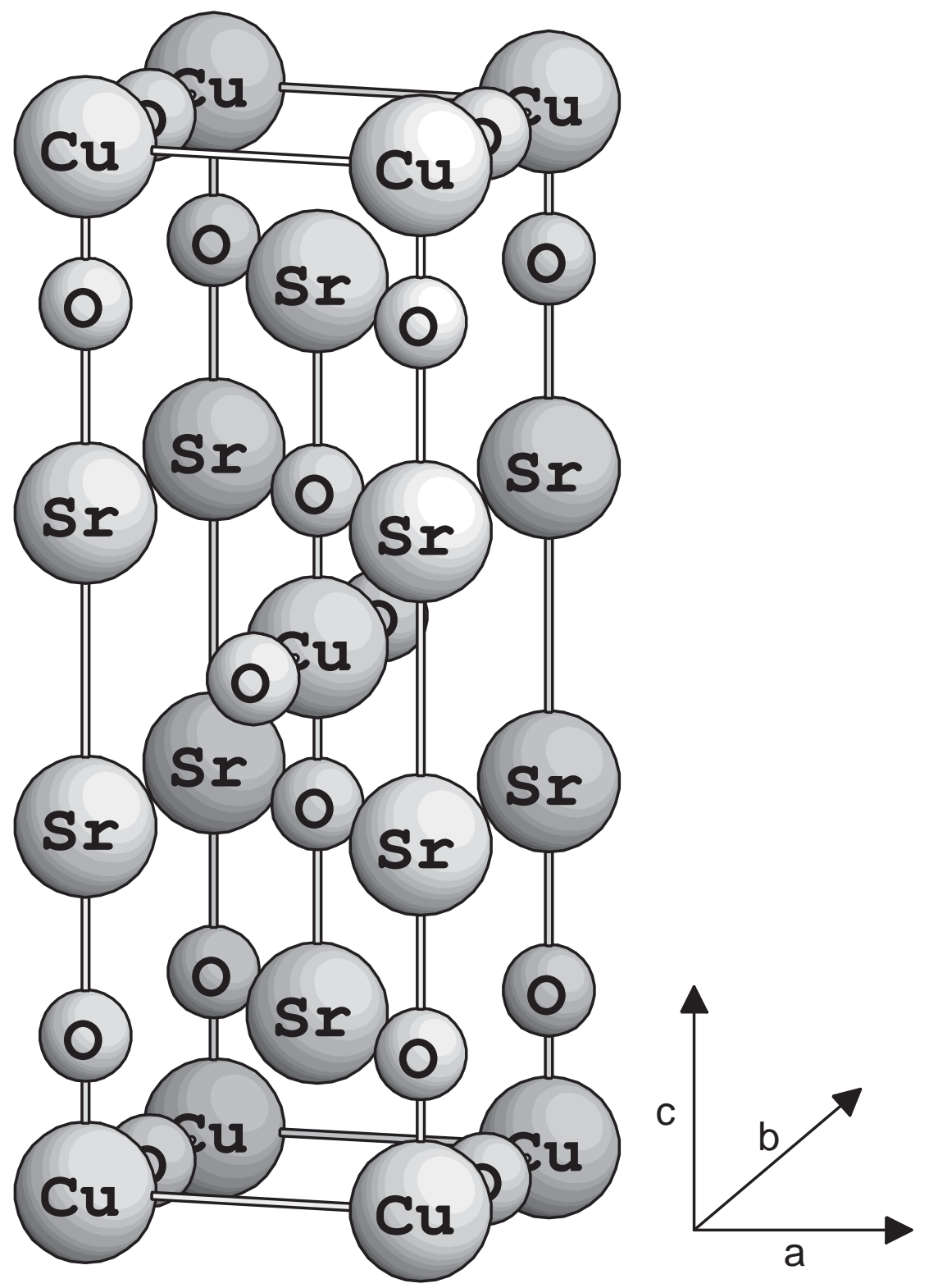




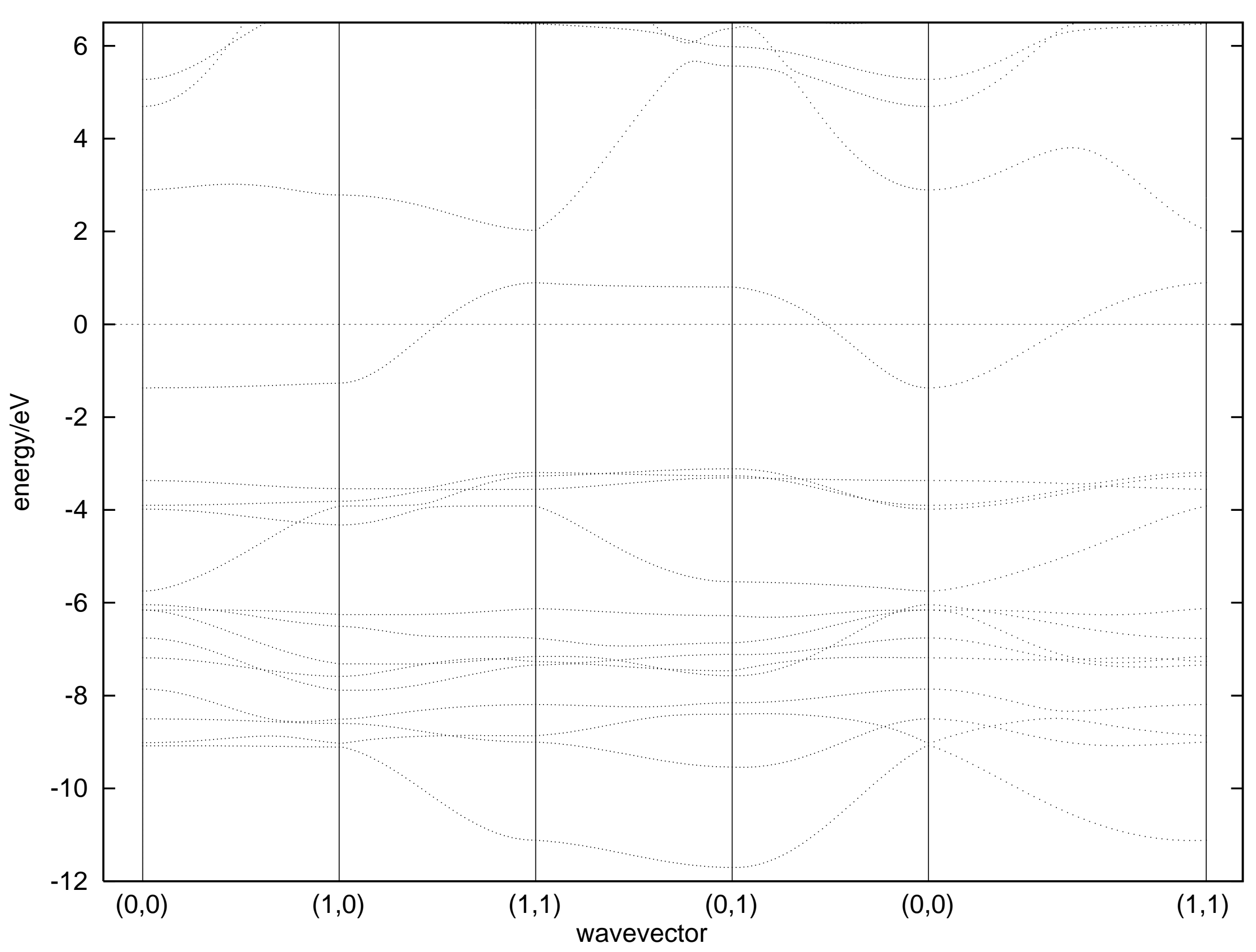

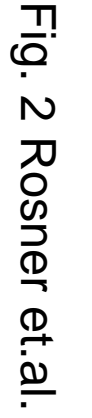




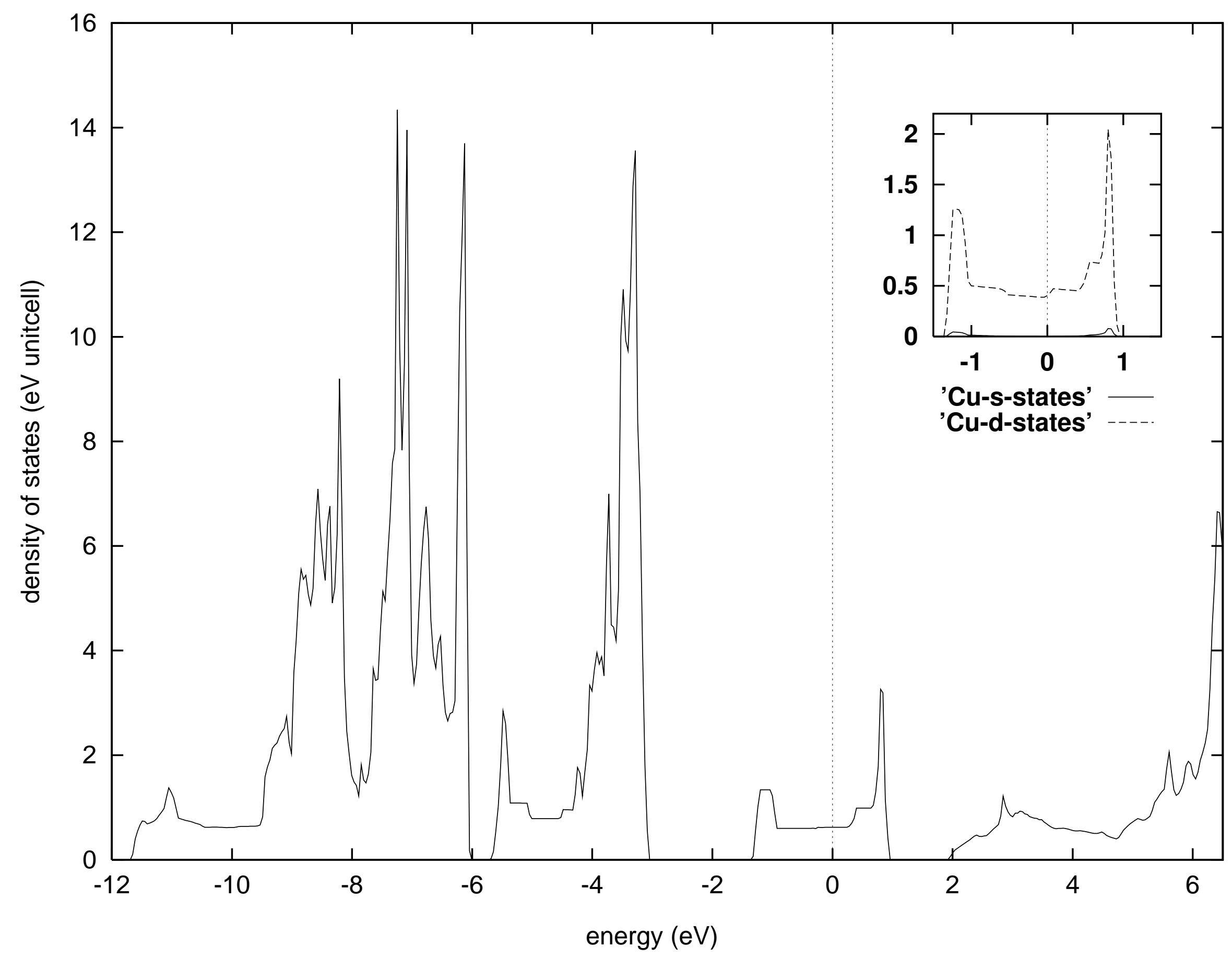

$\frac{\pi}{0}$

$\omega$

ग

只

$\stackrel{\Phi}{+}$

$\cong$ 


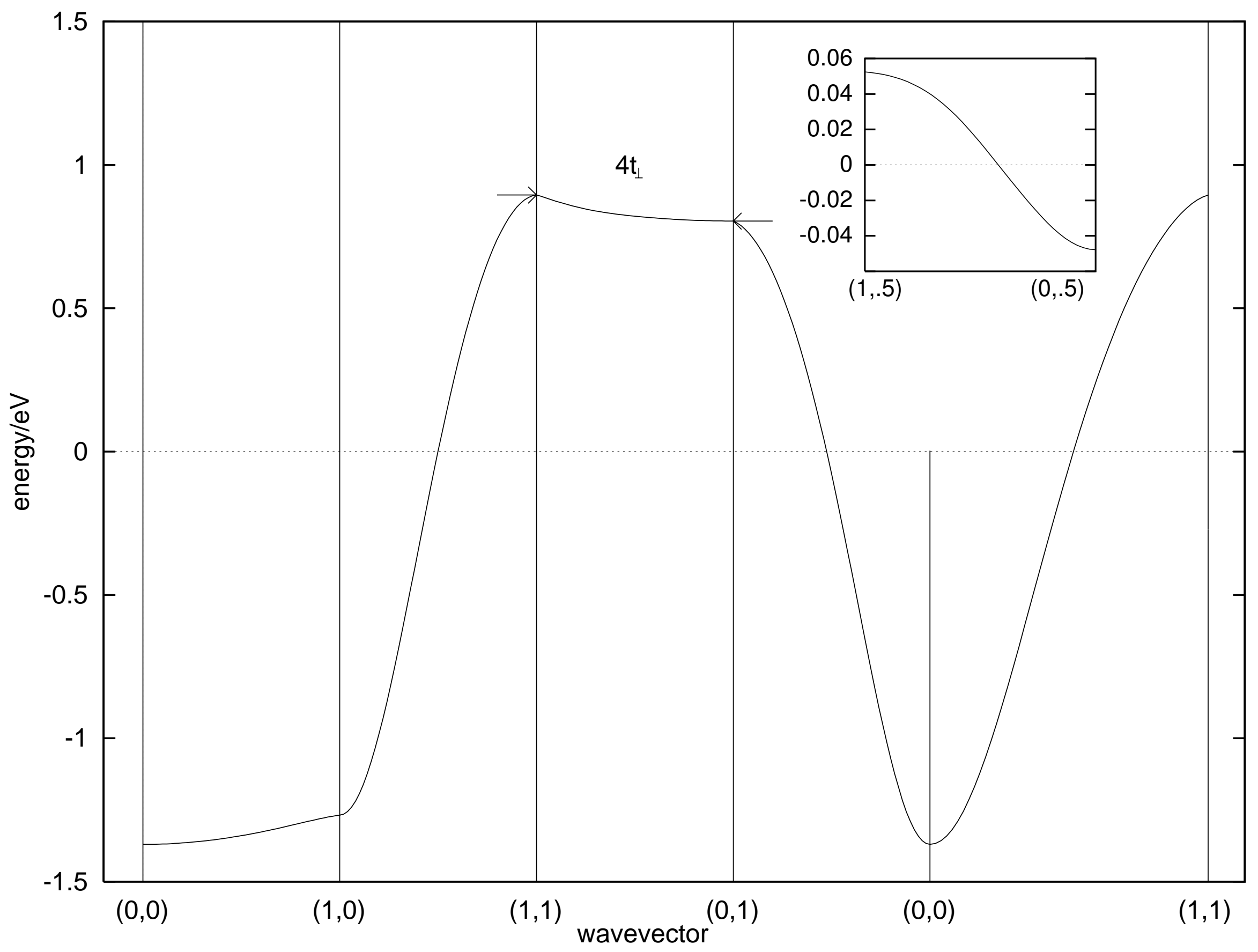

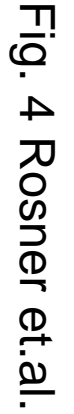



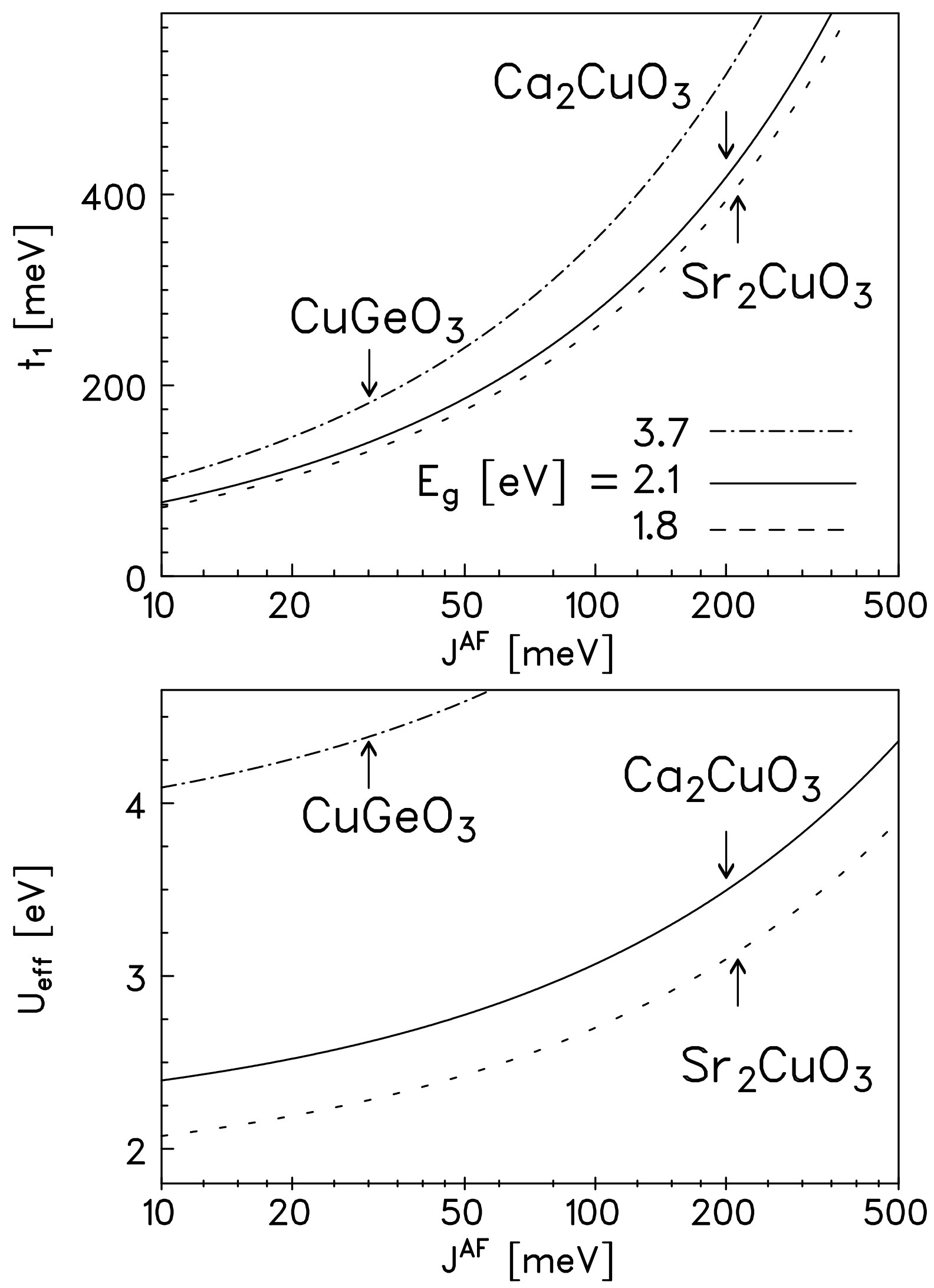

Fig. 5

Rosner et al. 